\title{
Cluster Phase Reactions: Alkylation of Triphosphate and DNA Anions with Alkylammonium Cations ${ }^{\dagger}$
}

\author{
Robert Hodyss, Heather A. Cox, and J. L. Beauchamp* \\ Noyes Laboratory of Chemical Physics, California Institute of Technology, Pasadena, California 91125
}

Received: June 9, 2004; In Final Form: July 12, 2004

\begin{abstract}
Electrospray ionization is used to create what are demonstrated to be highly reactive, metastable clusters of alkylammonium cations and triphosphate dianions. Collision-induced activation of the clusters leads to either $\mathrm{S}_{\mathrm{N}} 2$ or E2 reactions, resulting in the formation of an alkyltriphosphate or triphosphate anion, respectively. The series of tetraalkylammonium ions from tetramethylammonium to tetraheptylammonium is studied. The $\mathrm{S}_{\mathrm{N}} 2$ pathway is favored over the E2 pathway. The replacement of a proton in the triphosphate dianion with a sodium cation in these clusters leads to enhanced yields of the E2 product. PM5 calculations of the reaction energetics correlate well with the observed product distributions. The doubly deprotonated DNA oligomer CCGG can also be alkylated by CID of its cluster with a tetraalkylammonium cation. The cluster phase reactions described in this study serve to illustrate a general scheme which we propose for the rational design of ionic clusters where reactivity is ensured by inhibiting direct dissociation. The experimental methodology implied by the cluster design criteria expands the utility of small molecular clusters for structure-reactivity studies and directed chemical synthesis.
\end{abstract}

\section{Introduction}

Molecular clusters, a bridge between the gas phase and bulk phases, provide a unique environment for structure-reactivity studies. However, relatively few investigations have focused on detailed studies of chemical reactions between cluster components. In part, this is due to the inherent difficulty of initiating reactions in clusters in competition with fragmentation of the cluster into its constituent monomers. In other words, activation results in the rupture of the weak noncovalent bonds that hold the cluster together rather than inducing reaction. In the present paper, we develop criteria for the design of clusters with latent reactivity, protected against dissociation, and then trigger reactions between the cluster components. This experimental methodology expands the utility of small molecular clusters for structure-reactivity studies and directed chemical synthesis.

A few groups have observed reaction between the components of molecular clusters held together by van der Waals interactions. Polymerization induced by light has been reported in clusters of carbon disulfide ${ }^{1}$ and in clusters of propene and benzene. ${ }^{2}$ Others have triggered reactions through electron impact. ${ }^{3}$ Reactions have also been initiated in clusters held together by ionic interactions. Beauchamp et al. produced ATP from clusters of the sodium salt of AMP. ${ }^{4}$ Gronert and co-workers extensively explored the reactions of alkylammonium cations with carboxylate anions in several pioneering studies. ${ }^{5-7}$ Williams and co-workers reported counterion substitution reactions in clusters of methonium cations and halide anions. ${ }^{8}$ These studies of ionic complexes all used collisional activation to initiate reactions.

Clusters with an inherent, latent reactivity can be created by considering a few basic design criteria. First, the cluster must

\footnotetext{
† Part of the special issue "Tomas Baer Festschrift".

* Author to whom correspondence should be addressed. E-mail: jlbchamp@caltech.edu.
}

be held together tightly enough to avoid fragmentation to monomers on excitation. This is accomplished by using the Coulombic attraction of ionic components. Ionic solids derive their stability from extended Coulombic interactions between oppositely charged ions. Even complexes of neutral molecules often derive stability from charged or partially charged functional groups within each molecule. For example, clusters of arginine in the zwitterionic form derive their stability from headto-tail interactions between the protonated guanidinium side group and the carboxylate., 9,10 Second, to ensure interesting chemistry, we also select systems with no possibility of the simple transfer of a labile proton that would lead to a weakly bound neutral component that is easily eliminated. Third, while it is not a general requirement, our mass spectrometric studies require that the cluster have a net positive or negative charge. In the simplest case, these criteria are realized by preparing binary clusters where one component carries two negative charges and the other carries a single positive charge. The polarity of the charges can be switched without loss of generality, and the number of cluster components and charged centers can be increased.

We report detailed systematic studies of reactions in anionic clusters of tetraalkylammonium cations and triphosphate dianions. Two reaction pathways are available: an $\mathrm{S}_{\mathrm{N}} 2$ pathway and an E2 pathway. These processes have distinct transition states, involving nucleophilic attack at the $\alpha$-carbon or the $\beta$-hydrogen of the tetraalkylammonium cation (Scheme 1). The contrasting energetics of these competitive reactions in solution and in the cluster are illustrated in Figure 1. The system begins in the solution phase (dashed lines), where both product channels are endothermic. PM5 calculations of the tetraethylammonium cation/triphosphate dianion sytem, using $\mathrm{COSMO}^{11}$ to model the solvation effects of water, show that the E2 reaction channel is favored by $\sim 170 \mathrm{~kJ} / \mathrm{mol}$ over the $\mathrm{S}_{\mathrm{N}} 2$ channel and that both processes are endothermic. Electrospray ionization generates the ionic cluster, with a salt bridge structure, in the gas phase (solid 
SCHEME 1: $\mathrm{S}_{\mathrm{N}} 2$ and E2 Reaction Pathways for the Tetrahexylammonium Cation/Triphosphate Dianion Cluster ${ }^{a}$

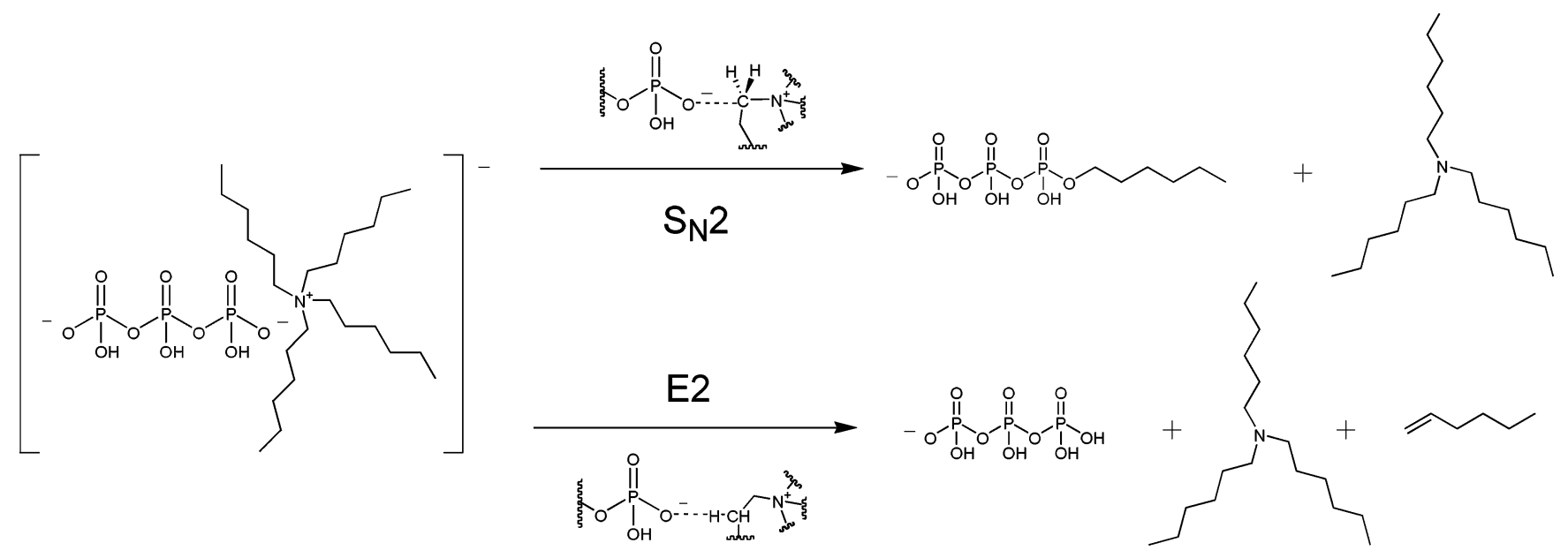

${ }^{a}$ Neutral products are inferred, not observed.

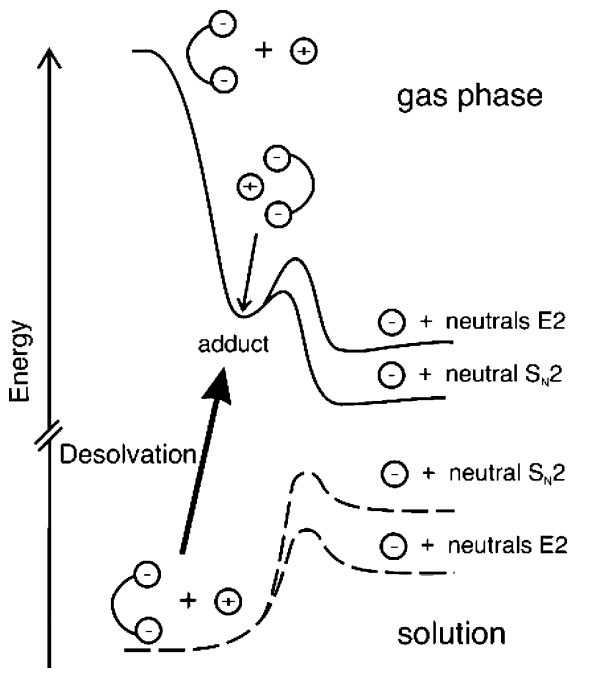

Figure 1. General energy-level diagram for the reaction of tetraalkylammonium ions with triphosphate dianion in the gas phase (solid lines) and the solution phase (dashed lines). Note that the energy scale is discontinuous.

lines). In the absence of solvent, dissociation of the cluster to the cation and dianion is highly endothermic, but the $\mathrm{S}_{\mathrm{N}} 2$ and E2 pathways are exothermic. The products are only loosely bound, comprising an anion and a neutral molecule. This combination of factors results in the double-well potential energy surface shown in Figure 1. In general, the $S_{N} 2$ pathway is of lower energy. In the cluster of doubly deprotonated tripohosphate dianion and tetraethylammonium cation, for the cluster to revert to reactants requires $523 \mathrm{~kJ} / \mathrm{mol}$. Formation of the E2 and $\mathrm{S}_{\mathrm{N}} 2$ products is exothermic by 50 and $150 \mathrm{~kJ} / \mathrm{mol}$, respectively. The relative heights of the reaction barriers are unknown, but both are probably on the order of $30 \mathrm{~kJ} / \mathrm{mol}$, similar to the barrier calculated for the analogous reaction of acetate with ethyltrimethylammonium. ${ }^{5}$

The energetics of this system highlight the differences in reactivity between the solution phase and the cluster phase. An endothermic reaction in solution may become exothermic when solvent is removed, and the product distribution may be substantially modified. The gentle desolvation process associated with electrospray ionization ${ }^{12,13}$ permits formation of a cluster which is metastable but does not have enough energy to surmount the barriers to product formation.

The series of tetralkylammonium ions from tetramethylammonium to tetraheptylammonium are investigated. Differ- ences in reaction behavior are observed when the asymmetric alkylammonium ions phenyltrimethylammonium and benzyltrimethylammonium are used. The replacement of a triphosphate proton with a sodium cation in these clusters increases the yield of the E2 product. We have also examined the reactions of tetraalkylammonium cations with multiply deprotonated DNA oligomers, resulting in alkylation of the DNA phosphate backbone.

\section{Experimental Section}

All experiments were performed on a Finnigan LCQ Classic ion trap mass spectrometer with an ESI source operating in the negative ion mode. The signal was tuned on the tetraethylammonium and triphosphate cluster using the automatic tuning capabilities of the instrument, and these parameters were used for all further experiments. Ions were subjected to collisional activation for $30 \mathrm{~ms}$. Relative product yields were measured as relative peak intensities in the mass spectrum. There may be small systematic variation in detection sensitivity at different masses, but this was not investigated. The activation conditions had little effect on the product ion distributions.

Samples were electrosprayed from methanol solutions using pentasodium triphosphate at a concentration of $50 \mu \mathrm{M}$ and alkylammonium bromide salts at concentrations between 50 and $75 \mu \mathrm{M}$. Solution flow rates were between 3 and $5 \mu \mathrm{L} / \mathrm{min}$. All compounds were purchased from Sigma-Aldrich and used without further purification.

Optimized structures and energies were calculated using the semiempirical PM5 method with CAChe 5.04 (Fujitsu, Beaverton, OR). The reaction energies are calculated from the energies of the bound reactant cluster and the separated product molecules. Gas-phase acidities were calculated using B3LYP/ $6-31 * * \mathrm{G}++$ with Jaguar 4.1 (Schrodinger, Inc., Portland, OR).

\section{Results and Discussion}

A. Symmetric Tetraalkylammonium Clusters. The negative ion mass spectrum in Figure 2a shows anionic clusters generated from a solution of pentasodium triphosphate and tetrahexylammonium bromide. Singly deprotonated triphosphate is evident at $257 \mathrm{~m} / \mathrm{z}$. A doubly deprotonated triphosphate/tetrahexylammonium cluster appears at $610 \mathrm{~m} / \mathrm{z}$, and the cluster composed of triply deprotonated triphosphate and two tetrahexylammonium cations appears at $963 \mathrm{~m} / \mathrm{z}$. Clusters in which one of the protons in the cluster has been replaced by a sodium cation are also 

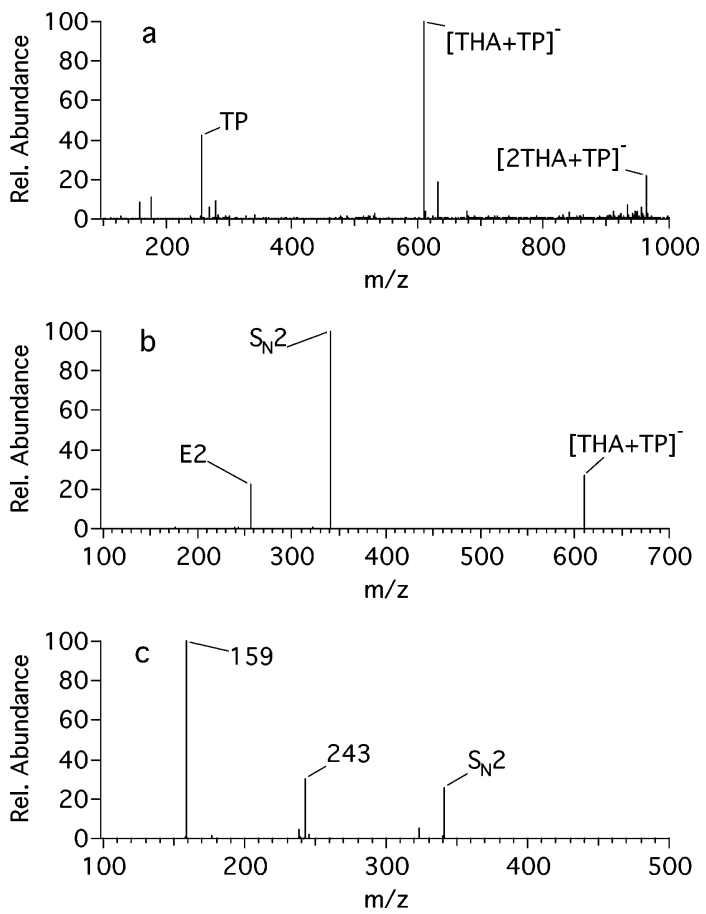

Figure 2. (a) Negative mode ESI mass spectrum of a solution of tetrahexylammonium (THA) bromide and sodium triphosphate. TP is triphosphate dianion or trianion. (b) Spectrum resulting from the collisional activation of the ion $[\mathrm{TP}+\mathrm{THA}]^{-}$from (a). (c) Spectrum resulting from the collisional activation of the $S_{\mathrm{N}} 2$ product from (b). See text for $\mathrm{m} / \mathrm{z}$ values of reactants and products.

generated. This spectrum is representative of the series of homologous alkylammonium cations examined, from tetramethylammonium to tetraheptylammonium.

The spectrum in Figure $2 b$ shows products resulting from collisional activation of the isolated cluster at $610 \mathrm{~m} / \mathrm{z}$. Instead of simple fragmentation of the cluster, collisional activation induces reaction between the cluster components according to Scheme 1. The two reaction pathways are observed include: an $\mathrm{S}_{\mathrm{N}} 2$ reaction that generates the hexyl triphosphate anion (341 $\mathrm{m} / \mathrm{z}$ ) and an E2 reaction that generates singly deprotonated triphosphate anion $(257 \mathrm{~m} / \mathrm{z})$. These reactions are analogous to those reported by Gronert et al. ${ }^{5-7}$ This reaction behavior is general for all the symmetric alkylammonium cations studied.

The $\mathrm{MS}^{3}$ spectrum of the $\mathrm{S}_{\mathrm{N}} 2$ product is shown in Figure $2 c$. The spectrum is entirely consistent with our assignment of the product as hexyl triphosphate. The fragment at $243 \mathrm{~m} / \mathrm{z}$ corresponds to a loss of $\mathrm{H}_{3} \mathrm{PO}_{4}$, and the fragment at $159 \mathrm{~m} / \mathrm{z}$ results from the loss of neutral hexyl phosphate. The intensities of the fragments suggests that alkylation occurs on the terminal oxygen atoms, as would be expected from charge separation in the triphosphate dianion. The lack of the E2 product in this spectrum indicates that its presence in the $\mathrm{MS}^{2}$ spectrum is not the result of collisional dissociation of hexyl triphosphate and that the $\mathrm{E} 2$ and $\mathrm{S}_{\mathrm{N}} 2$ pathways are concurrent with distinct intermediates. As illustrated in Scheme 1, the $\mathrm{S}_{\mathrm{N}} 2$ pathway occurs through nucleophilic attack of a triphosphate oxygen at an $\alpha$ carbon of the alkylammonium cation. The E2 pathway proceeds through abstraction of a $\beta$ hydrogen by a triphosphate oxygen from the alkylammonium cation.

The series of tetraalkylammonium ions from tetramethylammonium to tetraheptylammonium were each clustered with doubly deprotonated triphosphate and subjected to collisional activation. The percentage of $\mathrm{S}_{\mathrm{N}} 2$ product as a function of carbon number in the alkyl chain is plotted in Figure 3a. The percentage of $S_{N} 2$ product increases in general as the alkyl chain
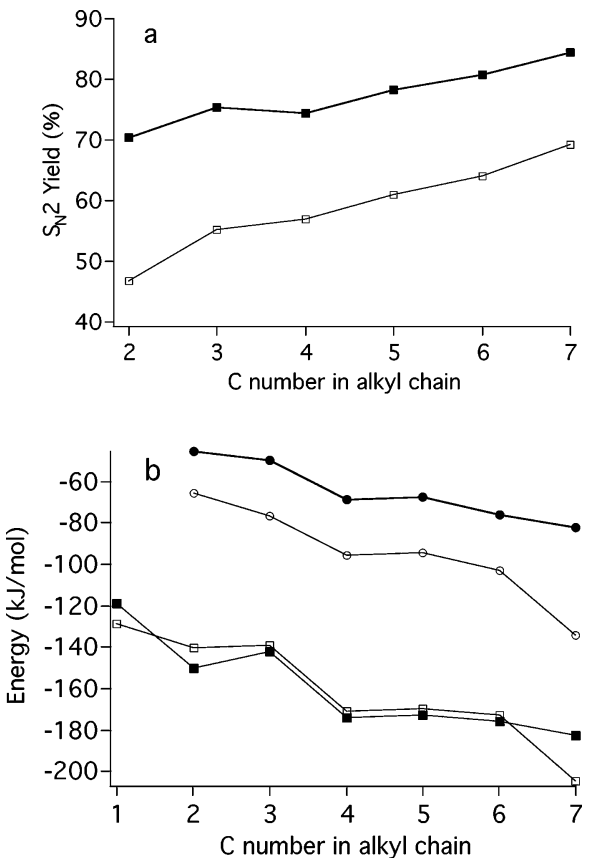

Figure 3. (a) Yield of $S_{N} 2$ product as a function of alkyl chain length of the tetraalkylammonium ion for protonated and sodiated clusters. Yields are typically reproducible to within $1 \%$. (b) Heats of reaction for $\mathrm{E} 2$ and $\mathrm{S}_{\mathrm{N}} 2$ reactions of triphosphate and tetraalkylammonium ions for protonated and sodiated clusters. Filled circles, E2; open circles, E2 for the sodiated clusters; filled squares, $S_{N} 2$; open squares, $S_{N} 2$ for the sodiated clusters.

lengthens. Figure $3 b$ gives the overall energetics of the $S_{N} 2$ and E2 reaction pathways as calculated by the PM5 method for each of the alkylammonium ions. The observation that the product distributions correlate well with the overall reaction energetics suggests that the barriers to reaction vary in the same manner with increasing chain length.

Clusters of triply deprotonated triphosphate and two alkylammonium cations can be activated mutiple times to induce multiple $S_{N} 2 / E 2$ reactions. For example, the spectrum resulting from collisional activation of the cluster composed of triply deprotonated triphosphate and two tetraheptylammonium cations shows the $\mathrm{S}_{\mathrm{N}} 2$ product, a cluster of doubly deprotonated heptylated triphosphate dianion and tetraheptylammonium cation, and the E2 product, a cluster of doubly deprotonated triphosphate and tetraheptylammonium cation. The yield of the $\mathrm{S}_{\mathrm{N}} 2$ product is $69 \%$, and the yield of the E2 product is $28 \%$. The remaining $3 \%$ is split evenly between products resulting from two $\mathrm{S}_{\mathrm{N}} 2$ reactions and an $\mathrm{S}_{\mathrm{N}} 2$ and an E2 reaction. The product of two $\mathrm{E} 2$ reactions, deprotonated triphosphate, was not observed, as it was below the mass range of the instrument. The yield of $\mathrm{S}_{\mathrm{N}} 2$ product is less than that obtained from the cluster of doubly deprotonated triphosphate and tetraheptylammnium cation (84\%). However, the yield is the same as that obtained from the sodiated version of this cluster (see Section C).

B. Phenyltrimethylammonium and Benzyltrimethylammonium Cations. The spectrum showing products from collisional activation of the cluster of phenyltrimethylammonium cation with doubly deprotonated triphosphate is given in Figure 4a. The only product observed is an ion at $271 \mathrm{~m} / \mathrm{z}$, corresponding to deprotonated methyl triphosphate anion. Neither the $\mathrm{S}_{\mathrm{N}} 2$ pathway (involving nucleophilic attack at an aromatic carbon) or a possible E2 pathway (yielding neutral benzyne) are observed with the phenyl substituent. 

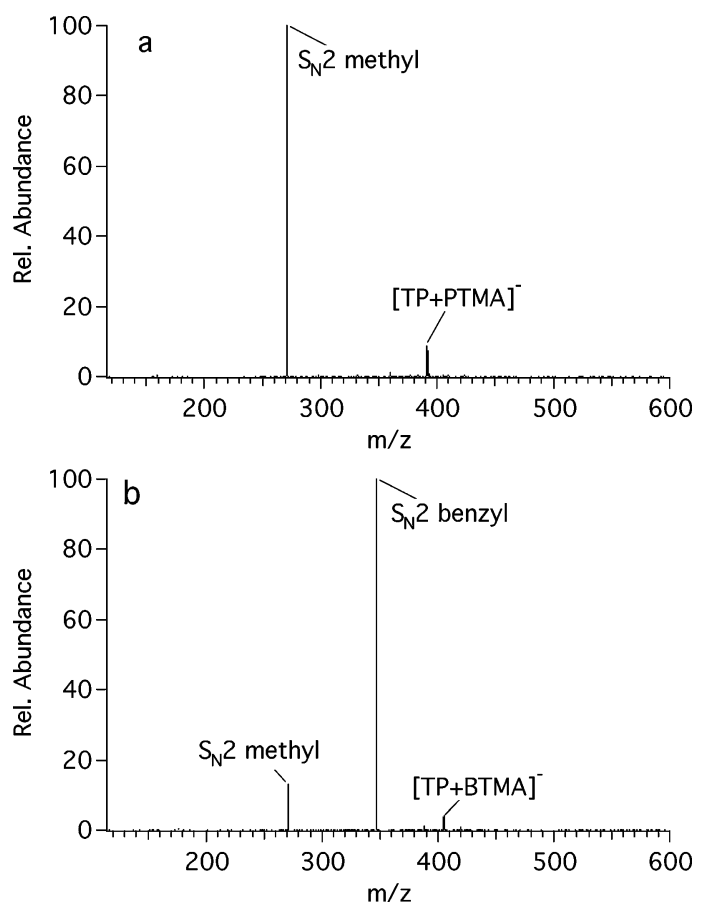

Figure 4. (a) Spectrum resulting from the collisional activation of the cluster of phenyltrimethylammonium cation (PTMA) with doubly deprotonated triphosphate (TP). (b) Spectrum resulting from the collisional activation of the cluster of benzyltrimethylammonium cation (BTMA) with doubly deprotonated triphosphate (TP).

Figure $4 \mathrm{~b}$ shows products resulting from collisional activation of a related cluster, benzyltrimethylammonium and doubly deprotonated triphosphate. In this case, $\mathrm{S}_{\mathrm{N}} 2$ reactions are observed involving both the benzyl and methyl substituents. The yield of the benzylated product is $88 \%$, despite the fact that the methyl product is statistically favored three to one. Gronert et al. noted the enhanced reactivity of the benzyl group in reactions of carboxylate with benzyltriethylammonium..$^{5}$ This is consistent with known trends in $\mathrm{S}_{\mathrm{N}} 2$ reactivity and can be attributed to the stabilizing effect of the phenyl substituent on the partial positive charge which develops on the carbon under attack.

C. Sodiated Clusters. The yields of $\mathrm{S}_{\mathrm{N}} 2$ products for the sodiated clusters are shown in Figure 3a. The results indicate that the replacement of a proton with a sodium cation increases the relative amount of E2 product produced. E2 yield is increased by $15-20 \%$ across the series of tetraalkylammonium ions, resulting in an inversion of the normal product distribution in the tetraethylammonium cluster, and so, for the tetraethylammonium cation, the E2 reaction is more favorable than the $\mathrm{S}_{\mathrm{N}} 2$.

A reasonable hypothesis for the enhancement of E2 reactivity on sodiation is that the replacement of a proton with a sodium cation increases the basicity of triphosphate, since strong bases favor the E2 reaction. ${ }^{14}$ However, DFT calculations of the proton affinities of triphosphate dianion with and without a bound sodium cation do not support this explanation. The calculated proton affinity of the triphosphate dianion increases by only 6 $\mathrm{kJ} / \mathrm{mol}$ when the proton attached to the central phosphate oxygen is replaced with sodium. The enhancement in E2 reactivity instead must be due to structural effects within the cluster.

PM5 calculations also agree with experiment. Figure 3 compares the energies of reaction for the $S_{N} 2$ and E2 products of the sodiated clusters with the nonsodiated clusters. While the energies of the $S_{N} 2$ reactions are quite similar for the sodiated and nonsodiated clusters, the substitution of sodium makes the E2 reaction more exothermic by $40-60 \%$.
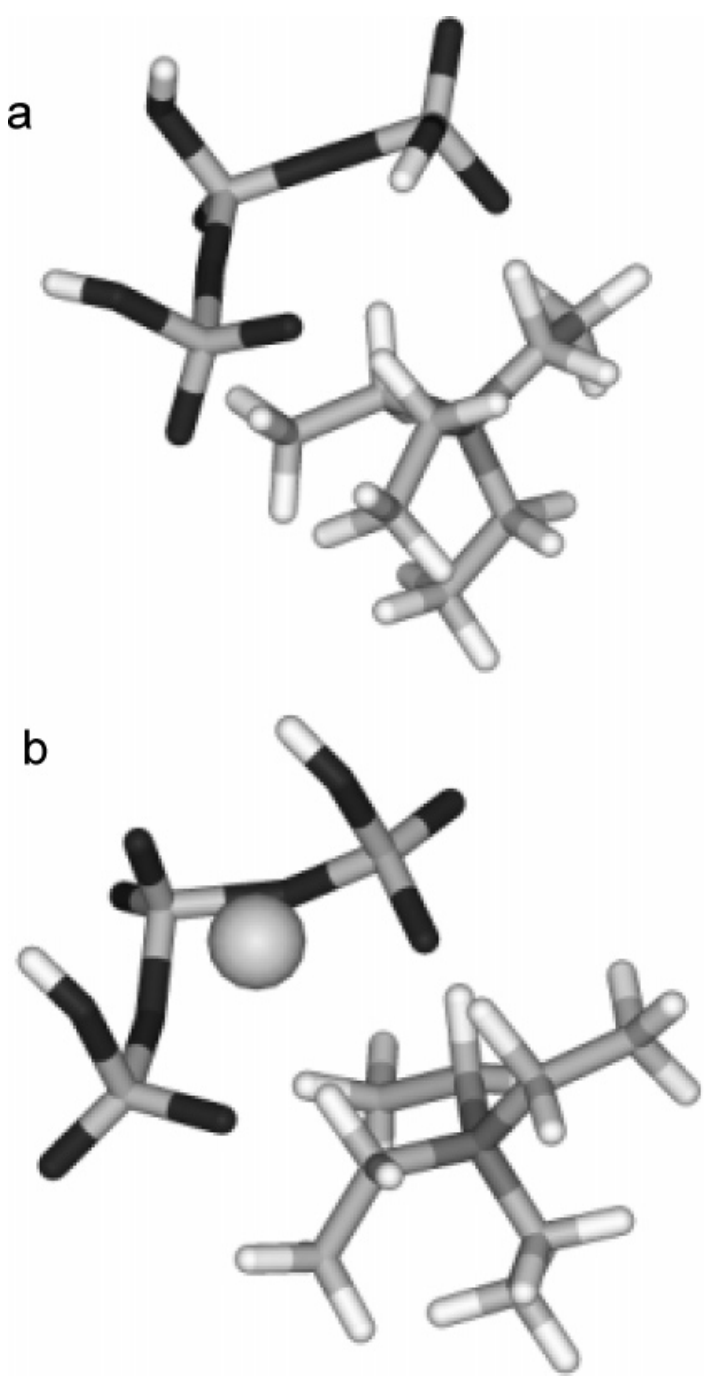

Figure 5. (a) Salt-bridge structure of the doubly deprotonated triphosphate dianion/tetraethylammonium cation cluster, calculated at the PM5 level. (b) Structure of the triply deprotonated triphosphate/ $\mathrm{Na}^{+}$/tetraethylammonium cation cluster, calculated at the PM5 level.

Figure 5 compares the structures of the sodiated and nonsodiated clusters of triphosphate and tetraethylammonioum cations, calculated at the PM5 level. In the cluster of doubly deprotonated triphosphate and tetraethylammonium cation (Figure 5a), the triphosphate dianion forms a salt-bridge-type structure with the cation. ${ }^{15}$ The deprotononated terminal oxygens both interact with the positive ammonium nitrogen. Figure $5 b$ shows the structure of the same cluster in which a triphosphate proton is replaced with a sodium cation. The tetraethylammmonium group is largely unaffected by the change, with the sodium cation interacting primarily with the neagatively charged oxygen atoms of the triphosphate.

D. Clusters with DNA. Other organic species containing phosphate groups should be amenable to reaction with alkylammonium ions. The phosphate backbone of DNA is a opportunistic choice, and tetraalkylammonium cations are known to cluster with DNA. ${ }^{16}$ Figure 6 a shows products resulting from collisional activation of the doubly deprotonated DNA oligomer CCGG/tetrabutylammonium cluster. The cluster $(1414 \mathrm{~m} / \mathrm{z})$ yields the expected products on activation: a butylated CCGG anion $(1229 \mathrm{~m} / \mathrm{z})$, the result of the $\mathrm{S}_{\mathrm{N}} 2$ reaction, and the CCGG anion $(1173 \mathrm{~m} / \mathrm{z})$, the result of the E2 pathway.

Figure $4 \mathrm{~b}$ shows products resulting from collisional activation of the $\mathrm{S}_{\mathrm{N}} 2$ product. The most prominent fragments are the $\mathrm{w}_{2}$ 

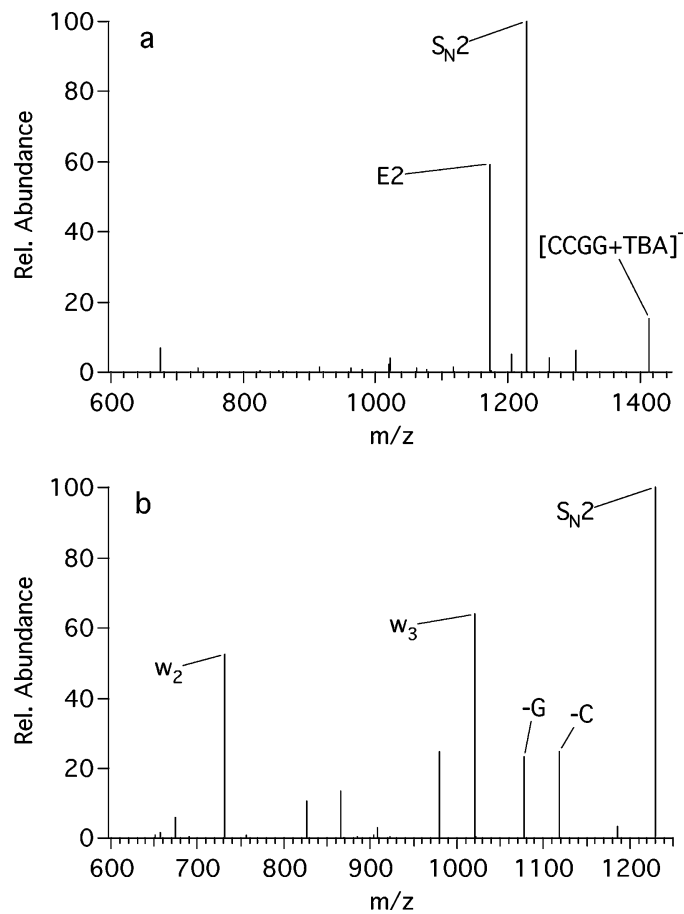

Figure 6. (a) Spectrum resulting from the collisional activation of a cluster composed of the doubly deprotonated DNA oligimer CCGG and tetrabutylammonium (TBA) cation. (b) Spectrum resulting from the collisional activation of the $\mathrm{S}_{\mathrm{N}} 2$ product from spectrum (a).

and $\mathrm{w}_{3}$ ions, both of which retain the butyl group. The w-type ions are formed by cleavage of a $3^{\prime} \mathrm{C}-3^{\prime} \mathrm{O}$ bond, yielding a fragment with a $3^{\prime}$ oligonucleotide end and a phosphate at the $5^{\prime}$ end. ${ }^{17}$ Loss of the bases cytosine and guanine is also present. We were unable to assign the remaining peaks to simple fragmentation processes. Collisional activation of the $\mathrm{S}_{\mathrm{N}} 2$ product does not yield a spectrum that positively identifies the site of alkylation. However, our results with alkylation of triphosphate suggest the site of alkylation is most likely on the phosphate backbone.

\section{Conclusion}

Tetraalkylammonium cations clustered with doubly deprotonated triphosphate anion undergo reaction when collisionally activated. Two reaction pathways are available: an $\mathrm{S}_{\mathrm{N}} 2$ reaction that produces alkylated triphosphate and trialkylamine and an E2 reaction that produces singly deprotonated triphosphate, a trialkylamine, and an alkene. The cluster of phenyltrimethylammonium cation with doubly deprotonated triphosphate reacts to yield deprotonated methyltriphosphate anion only. Reaction in the benzyltrimethylammonium cluster reveals that the reactivity of the benzyl moiety is much greater than that of the methyl. Sodiation of the clusters increases the proportion of E2 product. The reactivity of alkylammonium ions with triphosphate is consistent with known trends in reactivity and can be qualitatively modeled at the PM5 level.

The reactive clusters described here exemplify principles that can be used to design clusters with latent reactivity. Ionic bonds are used to hold the cluster together such that the addition of energy (through collision) does not fragment the cluster into monomers. We ensure that interesting chemical reactions will take place by creating clusters with no possibility of simple proton transfer that would lead to a weakly bound neutral component. The combination of a dianion and a cation in the cluster ensures that charged products will remain after reaction for mass spectrometric analysis.

Reactions within the cluster phase can exhibit behavior that is unlike that in the solution phase or the gas phase (Figure 1). Solution phase reaction of triphosphate and alkylammonium cations would lead to a product distribution different than that seen in the cluster phase, in which the E2 pathway is favored. The gas-phase energy surface contains a metastable state that can be accessed by a gentle desolvation process, such as electrospray ionization. The use of electrospray ionization to create metastable adducts is appealing as a method for the study of gas-phase reactivity since it provides access to the intermediate in gas-phase ion-ion reactions.

Modification of biomolecules in the gas phase is possible using reactive clusters, such as the alkylation of DNA demonstrated here. Alkylation of acidic residues and phosphorylation sites in peptides should be viable as well. The construction of macromolecular objects from large molecular clusters may also be possible. ${ }^{18}$

Acknowledgment. We thank Dr. Mona Shagoli for use of the Mass Spectrometry facility, as well as the Director's Research and Development Fund at the Jet Propulsion Laboratory and the Beckman Institute for funding. This material is based on work supported by the National Science Foundation under Grant No. CHE-0416381.

\section{References and Notes}

(1) Desai, S. R.; Feigerle, C. S.; Miller, J. C. J. Phys. Chem. 1995, 99 , 1786-1791.

(2) Pithawalla, Y. B.; Meot-Ner, M.; Gao, J.; El Shall, M. S.; Baranov, V. I.; Bohme, D. K. J. Phys. Chem. 2001, 105, 3908-3916.

(3) Garvey, J. F.; Peifer, W. R.; Coolbaugh, M. T. Acc. Chem. Res. 1991, 24, 48-54.

(4) Julian, R. R.; Beauchamp, J. L. Int. J. Mass. Spectrom. 2003, 227 (1), 147-159.

(5) Gronert, S.; Azebu, J. Org. Lett. 1999, 1, 503-506.

(6) Gronert, S. Acc. Chem. Res. 2003, 36, 848-857.

(7) Gonert, S.; Fong, L. M. Aust. J. Chem. 2003, 56, 379-383.

(8) Gross, D. S.; Williams, E. R. Int. J. Mass Spectrom. 1996, 158, $305-318$

(9) Julian, R. R.; Hodyss, R.; Beauchamp, J. L. J. Am. Chem. Soc. 2001, 123, 3577-3583.

(10) Julian, R. R.; Beauchamp, J. L.; Goddard, W. A. J. Phys. Chem. A 2002, 106, 32-34.

(11) Klamt, A.; Schuurmann, G. J. Chem. Soc., Perkin Trans. 2 1993, 5, 799-805.

(12) Smith, J. N.; Flagan, R. C.; Beauchamp, J. L. J. Phys. Chem. A 2002, 106, 9957-9967.

(13) Lee, S. W.; Freivogel, P.; Schindler, T.; Beauchamp, J. L. J. Am Chem. Soc. 1998, 120, 11758-11765.

(14) Saunders, W. H., Jr. Ionic Aliphatic Reactions; Prentice-Hall: Englewood Cliffs, NJ, 1965.

(15) Lee, S. W.; Kim, H. S.; Beauchamp, J. L. J. Am. Chem. Soc. 1998, 120, 3188-3195.

(16) Matulis, D.; Rouzina, I.; Bloomfield, V. A. J. Am. Chem. Soc. 2002, $124,7331-7342$

(17) McLuckey, S. A.; Habibi-Goudarzi, S. J. Am. Chem. Soc. 1993 , $115,12085-12095$.

(18) Zubarev, E. R.; Pralle, M. U.; Li, L.; Stupp, S. I. Science 1999 $283,523-526$. 